\title{
Simulating night visual conditions during the day with light attenuating filters
}

\author{
JOHN P. FARRELL \\ U.S. Army Research Institute for the Behavioral and Sucial Sciences, 1300 wilson Boulevard, Arlington. Virginia 22209
}

\begin{abstract}
This paper describes a research technique used to simulate night visual conditions during the day with light-attenuating filters. Some of the considerations in choosing light-attenuating techniques such as attenuation of infrared and ultraviolet illumination, neutrality, and clarity are discussed. A dual density filter with less attenuation in the lower field was chosen for aviation applications so that the major instruments would be available. This dual density concept was found to be more successful for piloting than for navigation. A density of 5.1 was shown to simulate a bright clear night with a full moon; a density of 5.5 simulated a much darker night with no moon.
\end{abstract}

In many research situations involving both theoretical and applied problems, it is advantageous to simulate night visual conditions during the day. Some examples are research on obstacle avoidance, pilot and nagivation behavior, automobile driving, dark adaptation and the interaction of illumination available to the eye with circadian rhythms and other functions. Simulated night conditions provide greater safety (especially in aviation applications), increased flexibility in planning and design of experiments, and greater facility in measuring and recording behavior.

A relatively inexpensive technique of mounting neu. tral-density light attenuating filters in goggles makes it possible to simulate different illumination conditions during the day. [Porterfield. Self, Heckart. Hanoven, \& McKechnie (Note 1) used a similar technique to simulate twilight and sunset-sunrise conditions.] The rechnique described in this paper was developed initially for use with helicopter pilots and navigators at the Army Research Institute for the Behavioral and Social Sciences.

Various materials for attenuating light while provid. ing a clear image were investigated. Photochromatic materials which darken upon exposure to light were reject ed because they attenuate light well only in the visible region and transmit infrared and ultraviolet. Since the pupillary reflex responds to visible light, the dilated pupils of subjects would admit potentially harmful radiation with this material. Photographic silver film attenuates visible light as well as infrared and ultraviolet but produces light scatter, which results in foggy images. Some ARI research used collodial carbon dis. persed in plastic and Eastman Kodak Wratten No. 96 filters of collodial carbon dispersed in gelatin. The collodial carbon filters have the advantage of being neutral in color attenuation, which is essential in achiev.

The views expressed in this paper are those of the author and not necessarily those of the US Army Research Institute for Behavioral and Social Sciences. ing perceptual equivalence between simulated night illumination and actual night conditions.

The plastic filters containing collodial carbon supplied by Omnitech $C_{0}$. simulated some night conditions rather well: however, against the sun a hazy condition developed from light scatter. Extremely fine carbon particles. 14 Angstroms or smaller, must be used in this application to prevent light scatter. Kodak Wratten fijters use such fine-particle carbon but have some drawbacks. In addition to being somewhat fragile, Wratten nilters transmit an incressing amount of infrared beginning at about 1 micron. as measured on a Perkin-Elmer Model 350 Spectrophotometer; they attenuate ultraviolet and visible light well. By adding a layer of Scotchtint Solar Control Film to the filters (plastic film containing metallic particles that attenuate light by reflection) the transmission of infrared is effectively prevented.

Data reported in this paper were obtained using a sandwich of filters consisting of several elements. The neutral gray plastic lens for glare protection used in the military sun, wind, dust goggles manufactured by the American Optical Co. is the basic item to which the other elements are affixed. A layer of Scotchtint film is added to this. In the upper part of the goggle, two Wratten filters, one of a density of 3.0 and one of 0.5 , are cut from Kodak $250 \mathrm{~mm} \times 250 \mathrm{~mm}$ sheets and added. In the lower portion of the goggles, a Wratten filter of 2.0 density is used. A layer of clear acetate is added to the inside surface to protect these items. The total density of the resulting goggles was measured in a Cary Model 14 spectrophotometer and the readings integrated against the photopic visual function. The upper portion of the goggles have a density of 5.1 and the lower portion 3.5 .

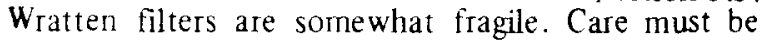
exercised to avoid scratching, and they must not be exposed to temperatures above $110^{\circ} \mathrm{F}$. (In the future. we plan to investigate the effectiveness of a direct coating of evaporated metal on plastic containing organic dyes to obtain a more durable filter.) 
In determining density of a goggle when a sandwich of filters is used, you can sum the densities of the individual filters only if absorption is the only attenuating technique used. Absorption filters obey the BouguerLambert law, i.e., the spectral transmittance of two or more optical filters used simultaneously is equal to the product of the spectral transmittance of each filter. Since density is equal to the log of 1 over transmittance, $\mathrm{D}=\log _{10} 1 / \mathrm{T}$, the densities of the filters involved can be summed (Note 2). Filters that attenuate by reflection do not obey the Bouguer-Lambert law and the density of the final filter combination must be measured.

The filters must be inserted in a comfortable goggle frame which forms a light-tight seal to the face. We have used two goggle frames; the standard military sun, wind, dust goggle manufactured by both American Optical Co. and Omnitech Co., and the Glendale Optical Co. Type 100 all-purpose goggle. Ventilation holes in the frames are made light-tight by gluing black foam rubber in the vents or using a black photographic tape over the vents.

Light-tight goggle frames cause perspiration, especially in warm weather. One solution that works well even with ambient temperatures of $80^{\circ} \mathrm{F}$ is to insert two dessicant bags supplied by Omnitech $\mathrm{Co}$. into each goggle. The dessicant bags are of black cotton cloth $5 \mathrm{in}$. long by $1 / 2$ in. in diam and contain silica. They are inserted between the frame and the lens over each eye and extend into the temporal area. After a few hours'use they must be reactivated by warming in a $250^{\circ} \mathrm{F}$ oven for $2 \mathrm{~h}$. Light-tight "zig-zag" vents and antifog coatings are currently being explored as additional approaches to the problem.

Natural daytime ambient illumination varies widely depending upon a large number of factors including: the presence or absence of clouds, the amount of water vapor in the atmosphere, the position of the sun in the sky, time of day, latitude, and season. Night illumination also varies widely depending upon similar factors including the phase and position of the moon, clouds, transmittance of the atmosphere, and various astronomical phenomena such as airglow. Because of the variations between different geographical locations, researchers in other areas may require slightly more or less density to achieve the same simulated level of illumination.

Reference data on natural illumination conditions both inside and outside the UH-1 helicopter are provided by Stowell and Poston (Note 3). Their data were collected on a Gamma 2020 photometer and illumination inside the cockpit was recorded at the instrument panel. The Stowell and Poston data are presented in Table 1; all readings are in footcandles.

Additional reference data on night illumination conditions were gathered by our laboratory at flight latitudes but at a distance of approximately 20 miles from the airfield to avoid contamination by airfield lighting systems. A Gamma 2020 photometer was used with a S-11 photocathode and a cosine filter to
Table 1

Illumination and the UH-I Helicopter

\begin{tabular}{lcc} 
Condition & \multicolumn{2}{c}{ Illumination Level } \\
Outside Cockpit & Inside Cockpit \\
\hline Daylight, Overcast & 1800 to 2300 & 400 to 800 \\
Full Moon, Clear & $1.25 \times 10^{-2}$ & $5.3 \times 10^{-3}$ \\
3/4 Moon, Clear & $3.7 \times 10^{-3}$ & $2.8 \times 10^{-3}$ \\
1/4 Moon, Clear & $3.3 \times 10^{-3}$ & $2.6 \times 10^{-3}$ \\
\hline
\end{tabular}

provide an integrated reading of the upper hemisphere in footcandles. Conditions were clear and moonlight readings were taken when the moon had risen to at least $40^{\circ}$ in the sky.

Flight data were collected in clear weather with occasional patches of clouds. A Spectra Minispotmeter manufactured by Photo Research Corp. was used to collect illumination data during the flights which were conducted at Ft. Rucker, Alabama. lllumination level was $1000 \mathrm{fL}$ as measured on grass-covered earth in the sunlight. When occasional clouds passed over, illumination dropped to $600 \mathrm{fL}$. During flight several measurements were taken of the aircraft's instrument panel with the spotmeter located level with the pilot's eye. The colors on the instrument panel vary from black to gray, so the readings ranged from 400 to $700 \mathrm{fL}$ when the instrument panel was in sunlight, and from 50 to $300 \mathrm{fL}$ when the panel was in shade.

Research in our laboratories indicated that a bidensty goggle with less density in the lower portion ${ }^{1}$ made it possible to read the larger and more important instruments. Night conditions were simulated through the upper portion of the goggle when the pilot looked outside the cockpit (see Figure 1). Testing several different densities with pilots and navigators resulted in our choice of a 5.1 density in the upper field and 3.5 in the lower portion. Pilots and navigators report that this configuration provides the perceptual equivalent of a full moon on a clear night. For simulating a much darker night (some stars but no moon) in ground-based applications, a density of 5.5 was used. In somewhat similar research, Porterfield et al (Note 1) used filters with densities of 4.1 and 3.1 ; the density of 4.1 simulated the lower end of Civil Twilight, when the sun is $6 \mathrm{deg}$ below the horizon, and a density of 3.1 simulated light levels at sunrise and sunset.

The light-attenuating goggles shown in Figure 1 were tested with four pilots on a number of aviation tasks. The aircraft were UH-1 helicopters with dual sets of controls. A rated pilot without goggles was stationed at the dual controls for purposes of safety. At the attenuation used, 5.1 over 3.5, pilots easily held a course and fol-

Table 2

Noctural Illumination Levels at Test Site

\begin{tabular}{lc}
\hline Condition & Illumination Range \\
\hline Full Moon & $4.8 \times 10^{-3}$ to $1.4 \times 10^{-2}$ \\
Half Moon & $4.4 \times 10^{-4}$ to $2.1 \times 10^{-3}$ \\
Starlight & $1.5 \times 10^{-4}$ to $2.8 \times 10^{-4}$ \\
\hline
\end{tabular}




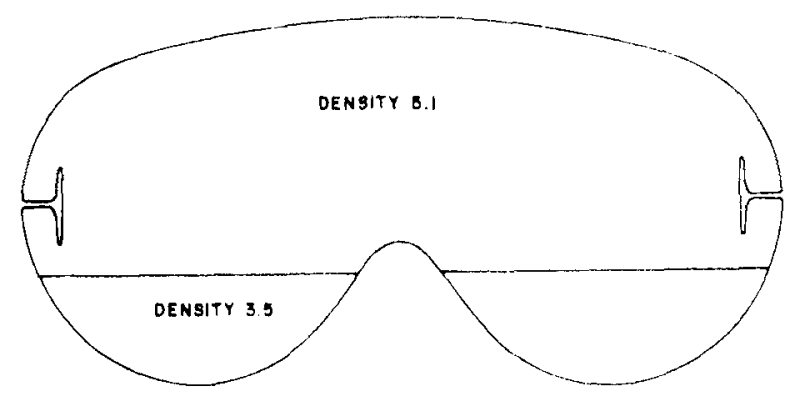

Figure 1.

lowed the directions of the navigating co-pilots. Pilots wearing goggles were able to fly in formation and to make routine maneuvers such as $360^{\circ}$ and $45^{\circ}$ bank turns without difficulty. In maintaining a stationary hover and in landing, the pilots tended to drift out of position, especially to one side or to the rear. The same drift problem while hovering or attempting to land arises in actual night helicopter flights.

In obstacle detection tasks, passing aircraft silhouetted against the sky could be detected at $500 \mathrm{~m}$. Telephone poles were detected at $60 \mathrm{~m}$ and wires at $45 \mathrm{~m}$ at slow aircraft speeds (below 40 knots) but not at higher speeds.

The dual intensity aspect of the light-attenuating goggles was somewhat less successful for navigation purposes. To a large extent, the ability to read maps and instruments depended on whether the aircraft was flying toward or away from the sun. When flying away from the sun, previously marked checkpoints on the map and major navigation instruments (such as the heading indi- cator, compass and windspeed indicator) were illuninated from the rear and were decipherable. When flying toward the sun, the map and instruments were in the shadow of the aircraft's dashboard and therefore too dark to be read.

Subjects report that the light-attenuating goggles provide a close simulation of actual night visual conditions. One exception to the generally close simulation was noted by two subjects; namely, when flying at night, ground lights such as street lights and automobile headlights are visible. Obviously, these were not present when night visual conditions were simulated.

A dual density goggle was selected for the aviation situation discussed here, but a single density may be appropriate for other uses.

\section{REFERENCE NOTES}

1. Porterfield, J. C., Self, H. C., Heckam, S. A., Hanoven, E. P., \& McKechnie, D. F. Airborne visual reconnaissance as a function of illumination level (AMRL-TR-9). Dayton, Ohio: Wright-Patterson Air Force Base. June 1971.

2. Eastman Kodak $\mathrm{Co}$. Neutral density attenuators. Kodak Publication No. P-114, 1971 .

3. Stowell, H. R., \& Poston, A. M. Phase I-An evaluation of current and potential instrument panel lighting techniques for use in Army helicopters. Technical Note 7-74. Human Engineering Laboratory, A berdeen, Maryland, A ugust 1974.

\section{NOTE}

1. The author is indebted to Dr. Aaron Hyman of the US Army Research Institute who suggested the bidensity concept for this application.

(Preceived for publication July 31, 1975; revision received October $6,1975$. 